\title{
Premature Ventricular Conduction Detection and Localization From the ECG Using a Neural Network
}

\author{
Alexander Pereira $^{1}$, Peter van Dam ${ }^{1,2}$, Roger Abächerli ${ }^{1,3}$ \\ ${ }^{1}$ Institute for Medical Engineering (IMT), Lucerne University of Applied Sciences and Arts, \\ Horw, Switzerland \\ ${ }^{2}$ Peacs BV, Nieuwerbrug, Netherlands \\ ${ }^{3}$ Cardiovascular Research Institute of Basel (CRIB), University Hospital Basel, Basel, Switzerland
}

\begin{abstract}
The identification and localization of premature ventricle contractions $(P V C)$ can be a lengthy procedure. In each treatment, a great deal of time is spent in the precise localization of the origin of the cardiac arrhythmia.

This work investigates the acceleration of the PVC detection process, using standard 12-lead ECG data as input and localizes $P V C$ on the right ventricular $(R V)$ or left ventricular $(L V)$. The proposed neural network (NN) is a shallow NN which consists of only one hidden layer with multiple hidden units. Three data sets consisting of a total of 328 resting ECG samples are used to train and evaluate the NN. Multiple iteration tests with different training sets have been done to identify the most promising configuration. The training cohorts differ in the distribution of data with PVC (cohort 1 ratio 1:1, cohort 2 ratio 25:4; NO PVC:PVC).

High sensitivity and specificity values have been reached in NNs with uniformly distributed training data providing a sufficient performance, which might be comparable to an expert.
\end{abstract}

\section{Introduction}

Premature ventricular conduction (PVC) occur in a large part of the population and are harmless to the healthy heart. 1-2\% of patients who have had a heart attack can develop ventricular cardiac arrhythmias, which can have life-threatening consequences. It can be treated by localization and ablation of the diseased tissue. The exact localization of the cardiac arrhythmia's origin can be a very time-consuming process, which, according to experience, can take two to twelve hours. Therefore, an automatic process should be developed to detect and localize the arrhythmogenic zone. This would speed up the diagnosis and keep the stress phase for the patient as short as possible. Moreover, it would also reduce treatment costs.

There is a trend towards approaches that are being made to automatize the ECG analysis such as arrhythmia detection in ECGs by using complex algorithms [1 - 11]. In addition, several algorithms are being developed in order to discover more detailed clinical pictures and to identify more specific arrhythmias, such as the VIVO method $[12,13]$. First results have been published which demonstrate the accuracy of PVC localization algorithms by comparing them against experts [14].

This work concentrates on developing a shallow neural network (NN), which can identify PVCs and match their origin to the LV or RV. The NN will be able to read standard 12-lead ECGs as well as detect them on PVC and respond with the corresponding ventricle side.

\section{Materials and methods}

\subsection{Neural network}

The NN was developed in MATLAB (R2016b, Mathworks, Natick, USA), which has a toolbox especially for developing and creating NNs. This toolbox provided an easy interface to build up the NN by identifying the input and the desired output. This work aimed to create a simple solution. Thus, a shallow NN with only one hidden layer was used.

The input for the NN are ECG data. On the one hand standard 12-lead resting ECGs are used, on the other hand the independent 8-lead resting ECGs. Due to the simplicity of the shallow NN, the input is static. Therefore, each data point from each lead is simultaneously given as an input to the NN. In order to assure that all ECG data are consistent in their length and form, the NN takes only samples of 10 seconds as input. Consequently, the NN has, depending on the number of leads, 120 '000 input units (12-leads) or 80 '000 input units (8-leads). This number is the equivalent to the number of leads multiplied by the number of seconds and sampling frequency.

The output is divided into three sections: PVC on the 
RV, PVC on the LV and NO PVC. These are the three main categories in this work. The NN does not localize the PVC exactly, but rather indicates a probability where the arrhythmia originates from.

\subsection{Data and preparation}

This work uses standard 12-lead resting ECGs of a total of 328 patients with and without PVC. Each ECG sample is recorded with a sampling frequency of $1 \mathrm{kHz}$. The ECG data are gained from three different clinical data sets. The VESRamin data [14] set consists of 55 resting ECGs with PVC. The second data set, the CEBRiS data set, consists of 255 stress ECGs without PVC. The NN should only use resting ECGs, which is why only the first minute of each stress ECG sample was extruded. The last data set is the ALVALE data set which consists of 18 resting ECGs. The VESRamin and CEBRiS data sets are used as the derivation cohort and the ALVALE as the validation cohort. The gold standard derives from a visual inspection.

The NN takes static data as input. Consequently, the data have to be prepared to fit into the NN. Each ECG file has to be split into multiple samples of 10 seconds each. The information from the ECG is stored in a matrix after reading it into MATLAB with the dimensions $12 \mathrm{x}$ (seconds times sampling frequency). Therefore, each file is divided into many 12 x 10,000 matrices, depending on their length. This resulted in a higher number of samples for the training and validation of the NN. The final number of samples in the VESRamin data set was 466, in the CEBRiS 970 and ALVALE 579. The distribution of the data samples with PVC incident is not 0.5 to 0.5 , except the VESRamin data set, which has an approximately even distribution of data samples with PVC and without PVC. The CEBRiS data set has no PVC occurrence in any sample. The independent 8-lead ECGs are generated by deleting the leads III, aVR, aVL and aVF from the matrix.

In a final step each matrix has to be transformed into a vector. This was handled by ranking each column from the matrix after the other. In the end each sample was a column vector with the dimension 120’000 (12-leads) or 80’000 (8-leads).

\subsection{Iteration tests and training $\mathbf{N N}$}

A shallow NN consists of one hidden layer. However, the number of units can be changed to improve performance and output. Therefore, multiple iteration tests with different hidden units (HU) have been performed and compared. The comparison was made by calculating the sensitivity and specificity of the iterations. Four configurations with different data sets were used to find out any differences in the output result. The VESRamin data set was used twice for training, once with the 12-leads and once with the 8-leads ECG. The other two iteration tests were done with a combination of the VESRamin and CEBRiS data set (12- leads and 8-leads). The two data sets differ in the number of samples and PVC distribution. The VESRamin set consists of 466 samples with an even distribution. The combination has 1436 samples, but the distribution ratio is 25:4 (NO PVC: PVC).

Before the NN can deliver correct results, it has to be trained with the corresponding data sets. However, not the entire data set is used for the training. $60 \%$ of the data set is used for training, $30 \%$ for validation and $10 \%$ for the test phase. The data gets randomly distributed to the various sets of the training. The training phase is used to adjust the weights and bias of the NN. The validation phase checks the error rate of the NN to get the best performance. In the test phase no more values are changed, but data is passed through without any comparison to the gold standard. This method is used to check whether the NN has learned anything at all.

A hundred iterations were made for each configuration set-up. The iterations differ in the number of HUs. The starting number of HUs was five with an increase of five after each number with a resulting final number of 100 . For each HU number five iterations have been made. The repeated use of the same number of HUs is done because the data is randomly distributed among the different sets. This approach makes it possible to identify whether the distribution of the data has an effect on the training.

\section{Results}

The number of HUs has a big influence on the performance. Since it cannot be calculated, it has to be determined with tests. For this reason, several iterations were performed. In order to filter out the best value, the sensitivity and specificity were calculated for each main category.

The results of the iteration tests are depicted in figure 1. The NNs trained with a uniformly distributed data set have median sensitivity values of $69.3 \%$ (12-lead) and $71.7 \%$ (8-lead). Their median specificity values are 88\% (12-lead) and $90.6 \%$ (8-lead). In comparison, $\mathrm{NN}$ without uniform distribution provided sensitivity values of $7.1 \%$ (12-lead) and $7 \%$ (8-lead) and have median specificity values of 98.9\% (12- and 8-lead).

The sensitivity and specificity values regarding each category (PVC on RV, PVC on LV) show significant difference. In the uniformly distributed data sets the median sensitivity values for finding PVC on the RV is $73.3 \%$ (12-lead) and $75.8 \%$ (8-lead), while the median specificity is 84.7 (12-lead) and $88.7 \%$ (8-lead). On the LV sensitivity values are $22.2 \%$ (12-lead) and 34.9\% (8-lead), whereas specificity values are $98.5 \%$ (12-lead) and $98.5 \%$ (8-lead). The combination set-up shows sensitivity of 7.9\% (12-lead) and 6.7\% (8-lead) for PVC detection on the RV as well as specificity values of $99.1 \%$ (12-lead) and $98.9 \%$ 
A

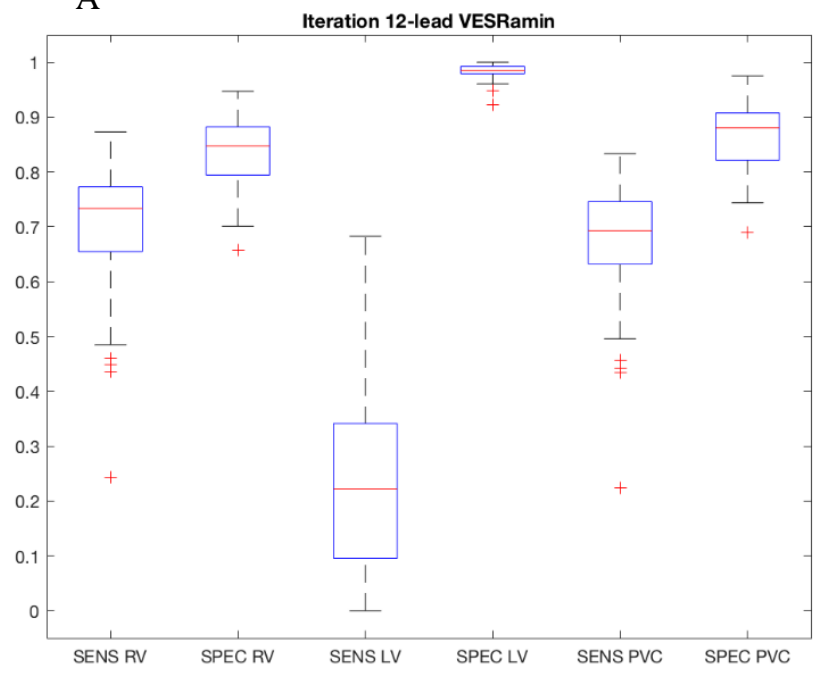

C

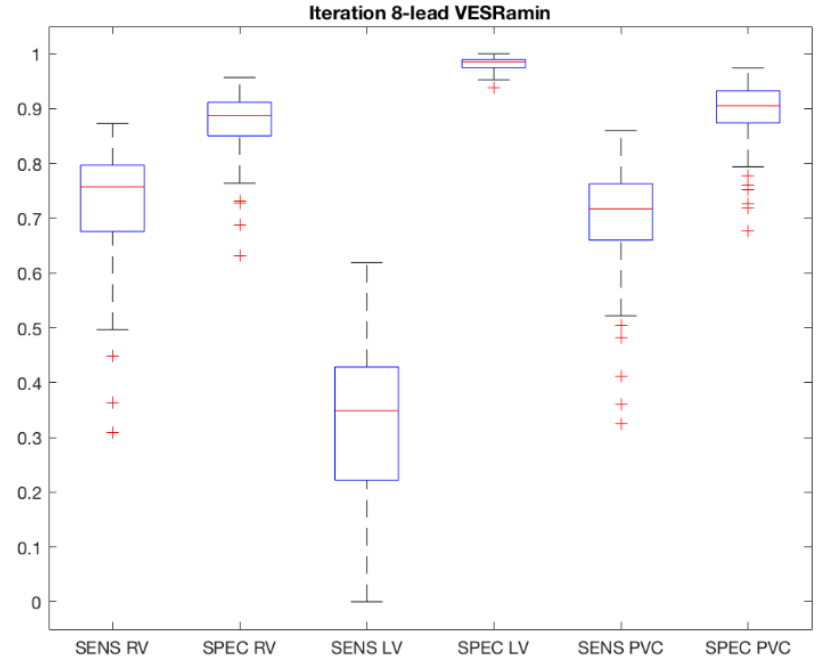

B

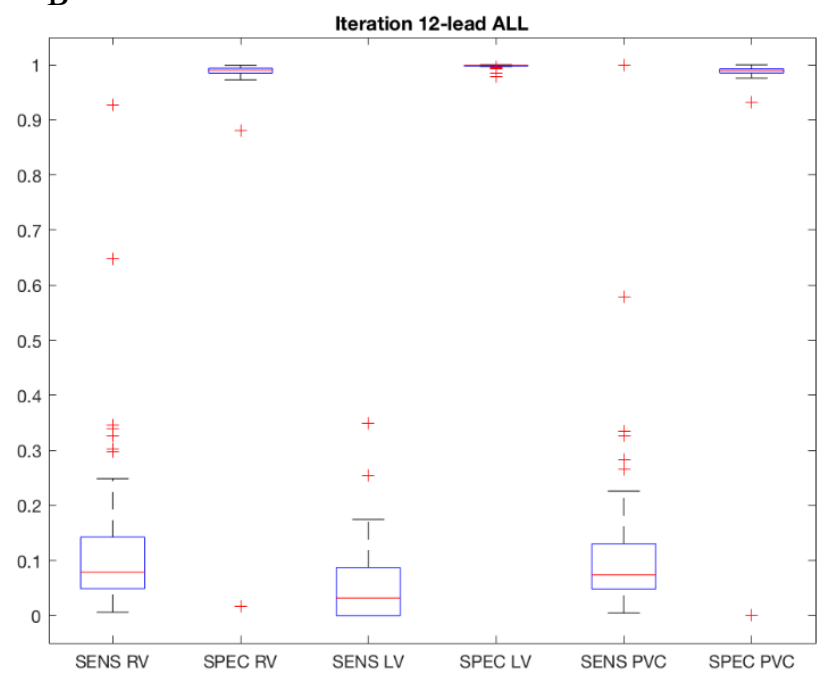

$\mathrm{D}$

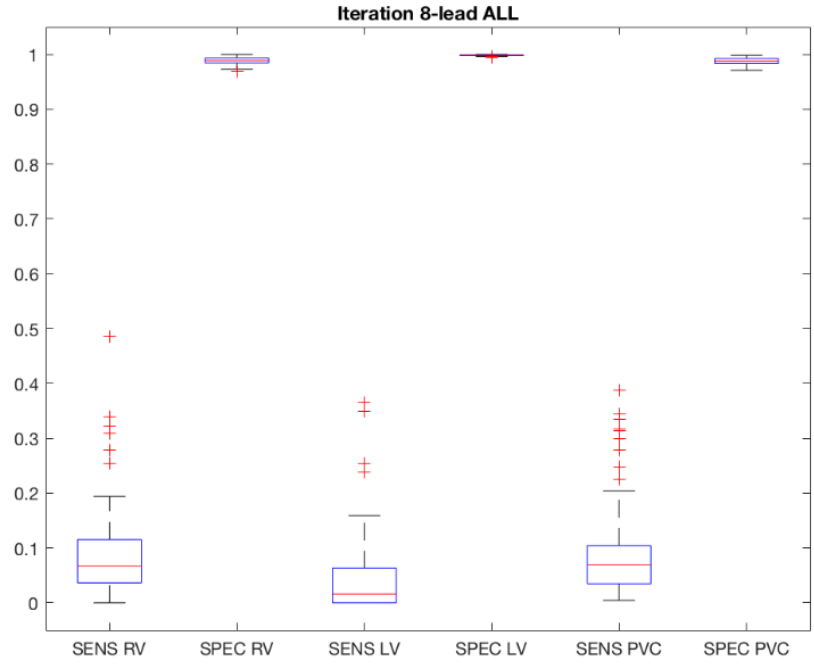

Figure 1. Boxplots of all the iteration tests A) VESRamin 12-lead B) Combination set 12-lead C) VESRamin 8-lead D) Combination set 8-lead

(8-lead). PVC detection on the LV resulted in sensitivity values of 3.2\% (12-lead) and 1.6\% (8-lead), and specificity values of $99.9 \%$ (12-lead) and $98.5 \%$ (8-lead).

The best performance regarding sensitivity and specificity was achieved with a $\mathrm{HU}$ number of 45 (VESRamin 12-lead), 100 (VESRamin, 8-lead), 75 (combination, 12-lead) and 85 (combination, 8-lead).

\section{Discussion}

There are two major points that have to be analyzed more in-depth. First, an analysis had to be conducted regarding the difference it makes when training the NN with two different training cohorts. Although the combination shows more data samples, the results are significantly worse compared to the VESRamin data set on its own. The sensitivity decreased significantly, but the specificity is very compact, close to $100 \%$. The reason for the large difference in values is the relatively smaller distribution of samples with and without PVC. In the VESRamin data set the distribution is almost $1: 1$, but in the combination only one in six is a case with PVC.

It is expected that there is hardly any difference between the results of the 12-leads and the 8-leads. When comparing the two methods, however, small differences can be seen. If the two box plots are placed next to each other, the shapes and distributions are similar, but on closer 
inspection it becomes apparent that the values shift by a few percent. From a purely mathematical point of view, the standard 12-leads can be calculated from the independent 8-leads. For this reason, the results should look identical. Since the single results depend on the arbitrary division of the data and the HU numbers, it is not inconceivable that differences can occur. Basically, they are small differences that could possibly be reduced with a higher number of iterations. Statistically speaking, the standard deviation would be reduced and therefore also the error between the two methods.

The results show a reasonably high sensitivity and specificity on the VESRamin data set. However, it should be analyzed if the NN is possibly overfitted. For this, the sensitivity and specificity were calculated for each phase of the training. In Table 1. can be seen how the sensitivity values decrease from the training to the test phase. Specificity, however, remains consistent. This pattern in the sensitivity values indicates overfitting of the NN.

Table 1. Sensitivity and Specificity values of a single iteration test in the VESRamin 12-lead training set.

\begin{tabular}{lllll} 
& Tot(\%) & Trai(\%) & Val(\%) & Test(\%) \\
\hline \hline SEN RV & 79.4 & 90.8 & 69.4 & 44.4 \\
SPE RV & 93.4 & 95.6 & $87.9 \mathrm{t}$ & 96.6 \\
SEN LV & 63.5 & 82.1 & 38.1 & 0 \\
SPE LV & 96 & 97.1 & 94.1 & 95.5 \\
SEN PVC & 81.6 & 92.7 & 72.9 & 38.1 \\
SPE PVC & 91.2 & 93.7 & 87.1 & 88.5 \\
\hline
\end{tabular}

\section{Conclusion}

It is a desirable goal to develop an algorithm to identify and locate PVC faster and more efficiently. This work showed a simple solution generated with the MATLAB toolbox. It is possible to locate PVC with the help of a shallow NN. The general sensitivity and specificity values could yield results with a similar accuracy as an expert, with the advantage that the NN delivers a constant performance [14].

\section{References}

[1] Badertscher P, et al. Automatically computed ECG algorithm for the quantification of myocardial scar and the prediction of mortality. Clinical Research in Cardiology 2018:107(9):824-835.
[2] Cupa J, et al. Diagnostic and prognostic value of QRS duration and QTc interval in patients with suspected myocardial infarction. Cardiology Journal 2018 Apr 3.

[3] Strebel I, et al. Diagnostic value of the cardiac electrical biomarker, a novel ECG marker indicating myocardial injury, in patients with symptoms suggestive of non-STelevation myocardial infarction. Annals of Noninvasive Electrocardiology 2018:23(4):e12538.

[4| Macfarlane PW, et al. Debatable issues in automated ECG reporting. Journal of Electrocardiology 2017:50(6):833-840.

[5] Krasteva V, et al. Biometric verification by cross-correlation analysis of 12-lead ECG patterns: Ranking of the most reliable peripheral and chest leads. Journal of Electrocardiology 2017:50(6):847-854.

[6] Jekova I, et al. Intersubject variability and intrasubject reproducibility of 12-lead ECG metrics: Implications for human verification. Journal of Electrocardiology 2016:49(6):784-789.

[7] Jekova I, et al. Inter-lead correlation analysis for automated detection of cable reversals in 12/16-lead ECG. Computer Methods and Programs in Biomedicine 2016:134:31-41.

[8] Krasteva V, et al. Real-time arrhythmia detection with supplementary ECG quality and pulse wave monitoring for the reduction of false alarms in ICUs. Physiological Measurement. 2016:37(8):1273-97.

[9] Reichlin T, et al. Advanced ECG in 2016: is there more than just a tracing?. Swiss Medical Weekly 2016:146:w14303.

[10] Krasteva V, et al. Superiority of Classification Tree versus Cluster, Fuzzy and Discriminant Models in a Heartbeat Classification System. PLOS ONE 2015:10(10):e0140123.

[11] Jekova I, et al. Threshold-based system for noise detection in multilead ECG recordings. Physiological Measurement 2012:33(9):1463-77.

[12] Misra S, van Dam PM, Chrispin J, Assis F, Kermaati A, Kolanaivelu A, Berger R, Tandri H. Initial validation of a novel ECGI system for localization of premature ventricular contractions and ventricular tachycardia in structurally normal and abnormal hearts. Journal of Electrocardiology 2018:51:801-808.

[13] van Dam PM. A new anatomical view on the vector cardiogram. The mean temporal-spatial isochrones. Journal of Electrocardiology .2017:50(6):732.

[14] Ebrahimi R, et al. Man vs. Machine: comparison of manual vs. Automated 12-lead ECG prediction of the origin of idiopathic ventricular arrhythmias to guide catheter ablation. European Heart Journal 2018:39:P4849.

Address for correspondence:

Roger Abächerli, Institute of Medical Engineering, T\&A, Lucerne University of Applied Sciences and Arts

Technikumstrasse 21, 6048 Horw, Switzerland

roger.abaecherli@hslu.ch 